\title{
Temporal Trends in Radiation Dose Associated with Coronary Computed Tomography Angiography
}

\author{
Paolo Marraccini ${ }^{1}$, Alessandro Mazzarisi ${ }^{1}$, Clara Carpeggiani1 ${ }^{1}$ Mathis Schluter ${ }^{2}$, \\ Marco Brambilla ${ }^{3}$, Massimiliano Bianchi ${ }^{4}$, Lorenzo Faggioni ${ }^{4}$, Giuseppe Coppini ${ }^{1}$, \\ Carlo Bartolozzi ${ }^{4}$, Eugenio Picano ${ }^{1}$ \\ ${ }^{1}$ Clinical Institute of Physiology, CNR, Pisa, Italy \\ ${ }^{2}$ CNR Tuscany Region G Monasterio Foundation, Pisa, Italy \\ ${ }^{3}$ Health Physics Unit, Ospedale Maggiore, Novara, Italy \\ ${ }^{4}$ Department of Radiology, Pisa University, Pisa, Italy \\ Email: *paolo.marraccini@ifc.cnr.it
}

Received 5 December 2013; revised 5 January 2014; accepted 12 January 2014

Copyright (C) 2014 by authors and Scientific Research Publishing Inc.

This work is licensed under the Creative Commons Attribution International License (CC BY). http://creativecommons.org/licenses/by/4.0/

(c) (i) Open Access

\section{Abstract}

Background: In 2010, the International Atomic Energy Agency launched the "3A's campaign" as an effective tool for primary cancer prevention. In 2011, the American Association of Physicists in Medicine recommended the size specific dose estimate (SSDE). Objectives: To audit doses of Coronary CT Angiography (Coronary CTA) in tertiary care referral center. Methods: We reviewed 998 consecutive Coronary CTA (from 2007 to 2012). Doses (CTDIvol mGy), DLP (mGy*cm), effective dose (DLP*0.014, mSv) were on-line archived. SSDE was estimated retrospectively. Appropriateness score was evaluated for exams performed from the 2010. Results: Overall median dose per Coronary CTA was $49.7 \mathrm{mGy}$ for CTDIvol, $55.5 \mathrm{mGy}$ for SSDE, $994.96 \mathrm{mGy}^{*} \mathrm{~cm}$ for DLP, $13.9 \mathrm{mSv}$ for effective dose. Median DLP decreased over time (1452.94 in 2007, 1605.56 in 2008, 1113.49 in $2009,759.99$ in $2010,448.61$ in 2011 and $497.88 \mathrm{mGy}^{*} \mathrm{~cm}$ in $2012, \mathrm{p}<0.0001$ ). SSDE was proportional to the size dependent factor (SDF); in patients with SDF > $1(88 \%)$ CTDIvol underestimated SSDE (48.49 vs $57.19 \mathrm{mGy}$ ), whilst in patients with SDF < $1(12 \%)$ CTDIvol overestimated SSDE ( 56.46 vs $50.3 \mathrm{mGy}$ ). Scans were appropriate in $58 \%$, uncertain in $24 \%$, and inappropriate in $18 \%$ of cases. Doses were similar in appropriate, uncertain or inappropriate examinations and in excellent-to-good $(81 \%)$ vs. sufficient-to-poor $(19 \%)$ image quality exams. Conclusions: Coronary CTA reference doses can be very misleading. SSDE can allow individual technique optimization. The dose is similar in appropriate and inappropriate examinations, and unrelated to image quality. The rate of inappropriate examinations is still too high even after dissemination of guidelines.

*Corresponding author. 


\section{Keywords}

\section{Ischemic Heart Disease; X Ray; Cardiac Computed Tomography; Cardiovascular Imaging; Radiation Dosing}

\section{Introduction}

The use of procedures with a high load of radiation has changed the practice of medicine, with unprecedented clinical and diagnostic benefits. Medical sources of radiation were about one-fifth that of natural radiation in 1987, close to half in 1993 and over 100\% that of natural radiation in 2006 [1], [2]. The contribution of CT in general, and Cardiac CT in particular, to cumulative radiation dose is high and still rising. In 2006, CT accounted for $24 \%$ of overall (natural and medical) radiation exposure, with nuclear medicine and interventional fluoroscopy accounting for $12 \%$ and $7 \%$ respectively [2]. The use of Cardiac CT is growing exponentially, and CT has been listed as a special focus of interest by the US President's Cancer Panel as an environmental cause of cancer amenable to reduction [3]. The process of radiological audit is essential to avoiding unnecessary medical radiation exposure in patients. In 2010 the International Atomic Energy Agency launched the "3A's campaign": audit, appropriateness, awareness for radiological justification and optimization, an effective tool for cancer prevention [4]. Optimization is especially important for Cardiac CT, whose doses may vary widely from less than $1 \mathrm{mSv}$ to more than $50 \mathrm{mSv}$ depending on the technology used, type of scan (with or without aorta), administration of contrast (with repeated scans and without contrast), patient habits and attention paid to optimization [5], [6]. This aspect is particularly important for cardiology patients, who already received high cumulative doses in the pre-cardiac CT era both as adult [7] and pediatric patients [8]. For these reasons, recently the Food and Drug Administration [9], American Heart Association [10], American College of Cardiology [11] and American College of Radiology [12] strongly recommended that each user facility, to a feasible extent, develop its own locally-based diagnostic reference levels, for use and audit until more broadly recognized levels are available. Moreover, in 2010 ACCF/SCCT/ACR/AHA/ASE/ASNC/NASCI/SCAI/SCMR published the "appropriate use criteria for cardiac computed tomography” [13], in 2011 the American Association of Physicists in Medicine, Task Group 204, provided an user-friendly method to estimate patient size specific dose (SSDE) [14] and Society of Cardiovascular Computed Tomography published the guidelines on radiation dose and dose-optimization strategies in cardiovascular CT [15]. All these tools foster justification and optimization balance. Aim of the present study is to audit the scanner radiation output and estimate of patient-size specific doses of cardiac CT over a 6-year period in a tertiary care cardio-radiology referral center.

\section{Methods}

In our institute, starting in 2007 we established an electronic database with a prospective archive of all invasive and non-invasive cardio-radiological examinations, linked with clinical records. In order to audit the Cardiac CT doses delivered in the last 6 years, we retrieved the data of 998 consecutive examinations performed in 998 patients (235 in-hospital and 763 outpatients, $63.3 \pm 11.7$ years, 669 males, 67\%, 329 females, 33\%), undergoing Coronary CTA. The examinations were performed at the CNR Tuscany Region G Monasterio Foundation from January 2007 to July 2011 and (due to an administrative transition) at the Radiology Department of the Azienda Ospedaliero-Universitaria Pisana from August 2011 to June 2012. Both Institutions used the same type of 64-slice machine (LightSpeed VCT 64, GE Healthcare, Milwaukee, WI, USA). The volume computed tomography dose index (CTDIvol, mGy) and the dose length product (DLP, $\mathrm{mGy}^{*} \mathrm{~cm}$ ), that represent the ionizing energy produced by scanner, were on-line recorded in the database. The effective dose $(\mathrm{mSv})$ was estimated by DLP-to-effective dose correction factor for chest in adults $(K=0.014)$ [16]. The effective patient size-specific dose (SSDE, mGy) was computed according to the following formula

$$
\text { SSDE }=\int_{\text {size }}^{32} \times \text { CTDI }_{\text {vol }}^{32}
$$

where $\int_{\text {size }}^{32}$ is a size dependent factor (SDF) that is a function of lateral and anterior-posterior chest dimensions [14]. These two parameters were retrospectively derived from the localizer images in each patient. 
For the 489 exams performed between 2010 and 2012, a clinician, generally a cardiologist, estimated, at the time of coronary CTA, the category indication and appropriateness score (from 1 to 3 inappropriate, 4 to 6 uncertain, 7 to 9 appropriate) by a computer-assisted calculator that facilitated the application of guideline rules [13] (Figure 1, Table 1).

Moreover, the radiologist, after the report, gave a subjective judgment of exam quality (insufficient, sufficient, good or excellent) according to the contrast enhancement of coronary arteries, presence of noise, moving artifacts and of the global readability of the images.

The Ethics Committee of the CNR Institute of Clinical Physiology approved the study. Each patient signed an informed consent that allowed us the use of the clinical information for this research.

\section{Statistical Analysis}

Continuous variables are expressed as median with interquartile range (IQR) and categorical variables as frequencies or percentages. We compared continuous data using no parametric tests such as Wilcoxon, KruskalWallis and Spearman where appropriate. We considered a p-value $<0.05$ statistically significant. We performed all statistical tests using the JMP package version 8.02 (JMP, Business Unit of SAS, SAS Campus Drive, Cary, NC 27513).

\section{Results}

Table 2 shows the clinical characteristics and CT dose in the overall population and patients grouped according to the CT technique. The values of DLP in 998 consecutive Coronary CTAs performed from 2007 to June 2012 are shown in Figure 2, where arrows indicate the introduction of a new acquisition technique. Over time, therewas a progressive decline of CTDIvol from 72.65 (30.2) mGy in the 2007 series to 24.89 (18.7) mGy in the 2012 series ( $<$ < 0.0001); effective dose showed a similar trend decreasing from 24.70 (10.3) to 8.46 (6.4) mSv in the same period. The first key factor affecting the dose was the decision to adopt the $100 \mathrm{Kv}$ instead of the

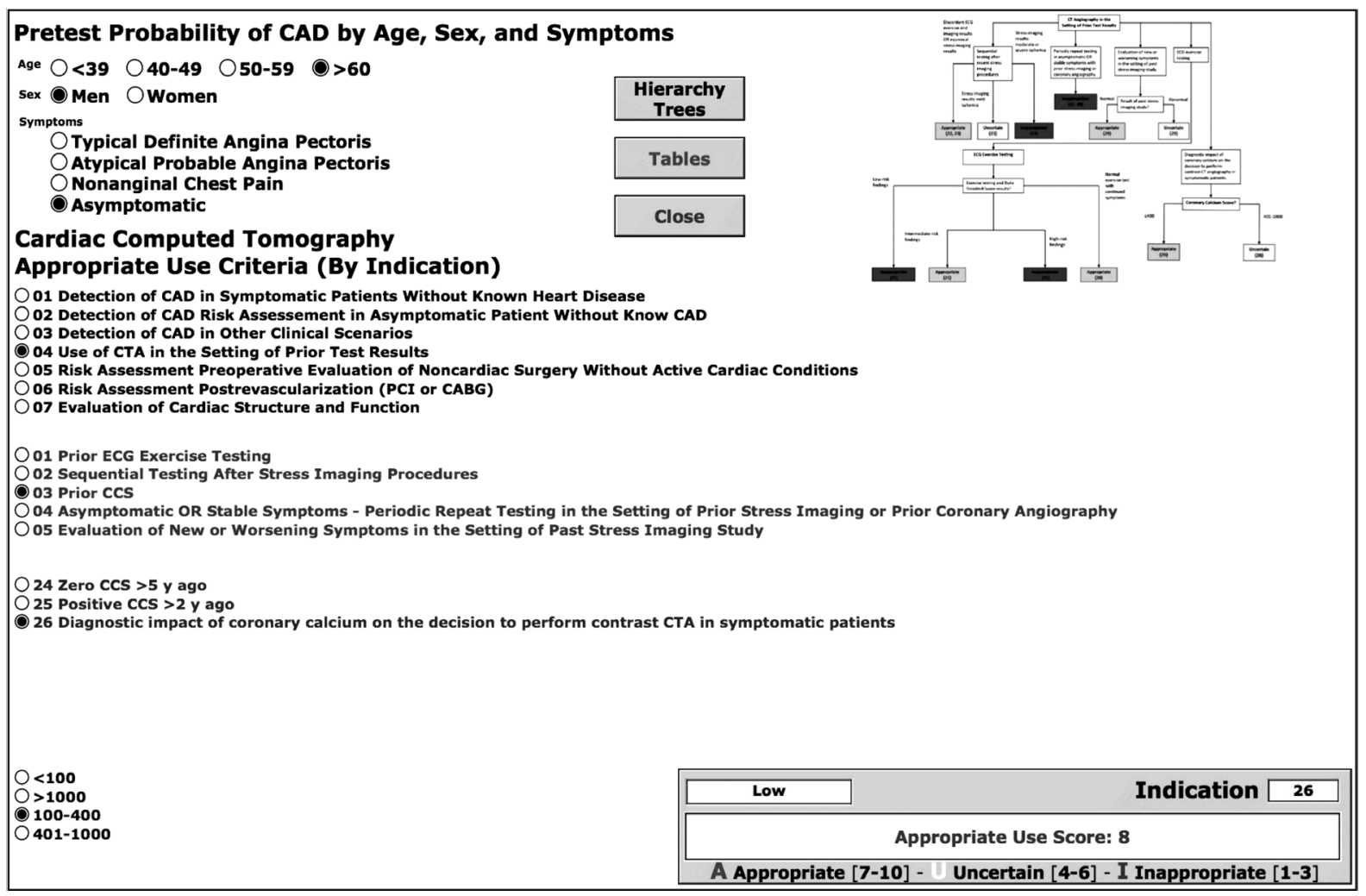

Figure 1. The user interface of the survey system for computing indication and appropriateness of cardiac CT examinations in accordance with the American College of Cardiology Foundation guidelines of 2010. 
Table 1. Clinical scenarios.

1) Detection of CAD in Symptomatic Patients Without Known Heart Disease

2) Detection of CAD/Risk Assessment in Asymptomatic Patients Without Known CAD

3) Detection of CAD in Other Clinical Scenarios

4) Use of CTA in the Setting of Prior Test Results

5) Risk Assessment Preoperative Evaluation of Non-cardiac Surgery Without Active Cardiac Conditions

6) Risk Assessment Post-revascularization (PCI or CABG)

7) Evaluation of Cardiac Structure and Function

Table 2. Clinical features and radiological parameters.

\begin{tabular}{|c|c|c|c|c|c|c|c|}
\hline & \multicolumn{4}{|c|}{ Patient groups according to $\mathrm{CT}$ technique } & \multicolumn{3}{|c|}{ Statistical analysis } \\
\hline & All & Spiral 120 kV (1) & Sipral 100 kV (2) & Axial (3) & 1vs2 & $1 \mathrm{vs} 3$ & 2vs3 \\
\hline \# (\%) & $998(100)$ & $573(57.4)$ & $308(30.9)$ & $117(11.7)$ & - & - & - \\
\hline Age (years) & $65(16)$ & $64(17)$ & $66(16)$ & $63(14)$ & ns & ns & ns \\
\hline Sex: M/F \# (\%) & $669 / 329(67 / 33)$ & $387 / 186(68 / 32)$ & 203/105 (66/34) & $79 / 38(68 / 34)$ & ns & ns & ns \\
\hline BMI & $26.3(4.7)$ & $26.6(4.99)$ & $25.6(4.61)$ & $26.7(6.1)$ & $\mathrm{p}<0.001$ & ns & ns \\
\hline Heart rate (bpm) & $58(11)$ & $60(12)$ & $60(14)$ & $55(12)$ & ns & ns & ns \\
\hline Sistolic blood pressure (mmHg) & $140(29.7)$ & $140(30)$ & $140(25)$ & $135(30)$ & ns & $\mathrm{p}<0.02$ & ns \\
\hline Diastolic blood pressure (mmHg) & $80(14.2)$ & $80(14.2)$ & $80(10)$ & $75(10)$ & ns & ns & ns \\
\hline DLP (mGy $\left.{ }^{*} \mathrm{~cm}\right)$ & $995(832)$ & $1384.5(575.8)$ & $670.4(192.1)$ & 316.5 (149.9) & $\mathrm{p}<0.001$ & $\mathrm{p}<0.001$ & $\mathrm{p}<0.001$ \\
\hline CTDIvol (mGy) & 49.7 (41.6) & $69.2(40.1)$ & 35.5 (9.1) & 15.8 (7.5) & $\mathrm{p}<0.001$ & $\mathrm{p}<0.001$ & $\mathrm{p}<0.001$ \\
\hline SSE (mGy) & $55.6(47.5)$ & $81.1(49.9)$ & $40.9(14.8)$ & $18.4(8.3)$ & $\mathrm{p}<0.001$ & $\mathrm{p}<0.001$ & $\mathrm{p}<0.001$ \\
\hline EffectiveDose (mSv) & $13.9(11.7)$ & $19.38(11.23)$ & $9.38(2.69)$ & $4.43(2.81)$ & $\mathrm{p}<0.001$ & $\mathrm{p}<0.001$ & $\mathrm{p}<0.001$ \\
\hline
\end{tabular}

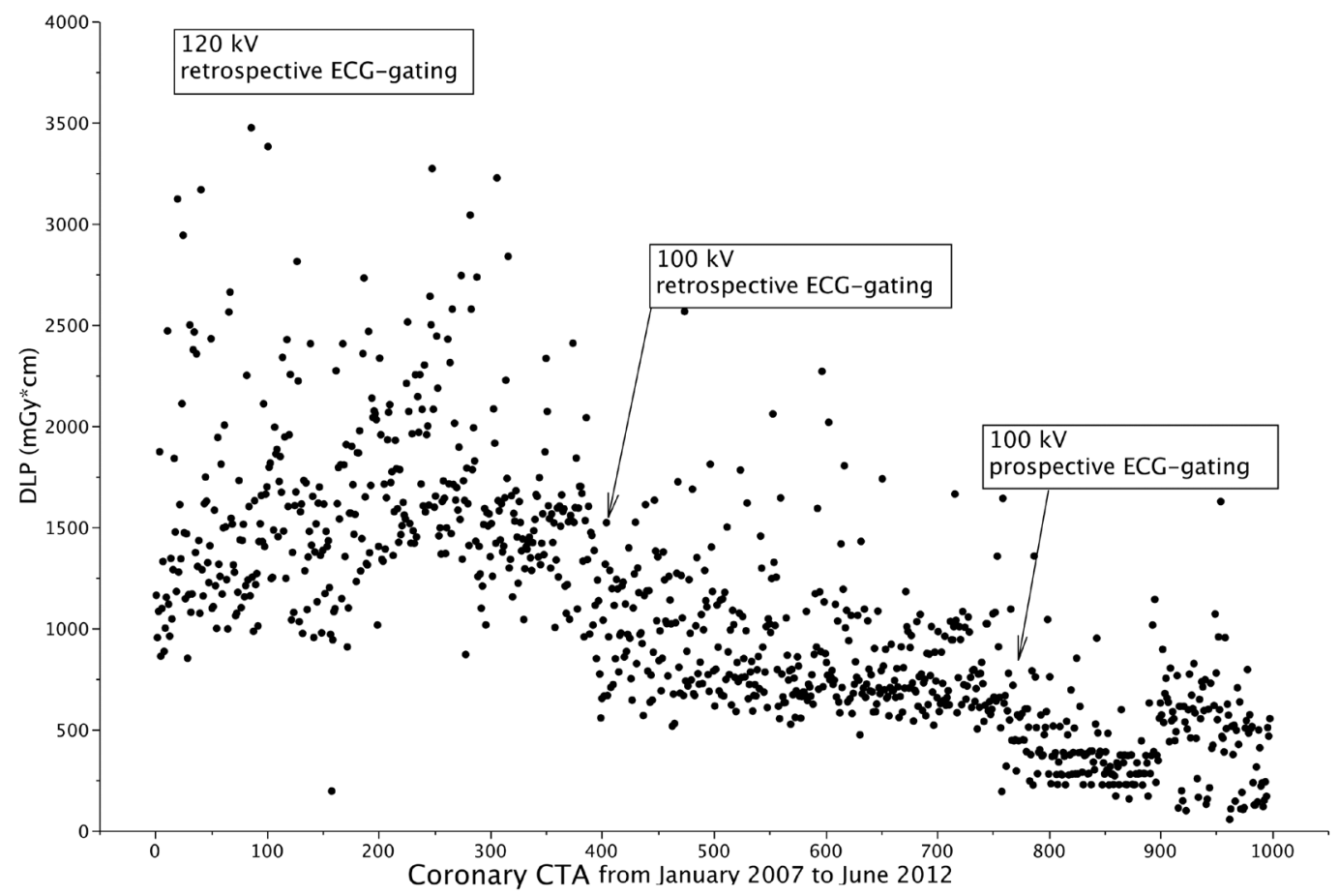

Figure 2. Time course of DLP in 998 coronary CTA examinations performed during the years 2007-2012. The arrows indicate the introduction of new techniques. 
$120 \mathrm{Kv}$ technique in patients with BMI < 30 (Table 2).

This technique allowed a reduction of the median effective dose from 19.38 (11.23) to 9.38 (2.7) mSv (p < 0.001). The second key factor was the use of axial acquisition with prospective ECG-gating in place of acquisition with retrospective ECG-gating, which allowed a decrease in the median effective dose from 9.38 (2.7) to $4.43(2.8) \mathrm{mSv}(\mathrm{p}<0.001)$.

In the 998 patients the median of SDF resulted $1.19(0.18), 88 \%$ of patients had a SDF $>1(1.23(0.18))$ and $12 \%$ of patients had SDF $\leq 1(0.95(0.11))$.

Figure 3 shows the temporal trends of CTDIvol and SSDE in these two groups of patients. In patients with SDF $>1$ the median value of CTDIvol and SSDE resulted 48.49 (41.9) and 57.19 (50.1) mGy, (p < 0.00001), respectively, the per-cent underestimation of SSDE by CTDI was $23(16) \%$. On the other hand, in patients with $\mathrm{SDF} \leq 1$ the median value of CTDIvol and SSDE resulted 56.46 (36.7) vs 50.3 (31.7) mGy (p < 0.00001$)$, and the percent overestimation of SSDE by CTDIvol was $5(11) \%$.

We retrieved data regarding clinical indications and appropriateness in the subset of 489 patients who underwent cardiac CT between 2010 and 2012. Based on the appropriateness score, we defined examinations as appropriate (58.3\%), uncertain (24.3\%) and inappropriate (17.4\%). In the group with uncertain indications, the prevalent score was 6 (67.2\% of the cases); i.e., the score tending toward appropriateness, with 5 and 4 scored by $11.8 \%$ and $21 \%$ of cases.

Figure 4 shows the distribution of the appropriate, uncertain and inappropriate exams in the clinical scenarios scheduled by guidelines (Table 1). The most frequent clinical indications were: "Use of CTA in the Setting of Prior Test Results" (scenario 4; 41.5\%), "Risk Assessment Post-Revascularization (PCI or CABG)” (scenario 6; 22.9\%), "Detection of CAD in Symptomatic Patients Without Known Heart Disease" (scenario 1, 19\%). The percentage of inappropriate tests was highest in scenario 2 (Detection of CAD/Risk Assessment in Asymptomatic Patients Without Known CAD) with 90.9\% rate (20 exams out of a total of 22), while ranging between 0 and $18.3 \%$ in the remaining clinical scenarios.

The quality of examination resulted excellent, good, sufficient and insufficient in $32.8 \%, 48 \%, 15.2 \%$ and $4 \%$ of examinations respectively. The dose administered to patients was not correlated with the degree of appropriateness nor with the quality of examinations performed (Figure 5 and Figure 6).

In the exams performed with retrospective ECG-gating and spiral technique, the use $100 \mathrm{kV}$ did not affect the quality score of examinations in comparison with the $120 \mathrm{kV}$ cases.

On the other hand, with the use of axial technique and prospective ECG-gating, as compared to the $100 \mathrm{kV}$ spiral technique, we observed an increase, although not-significant, in insufficient and sufficient examinations

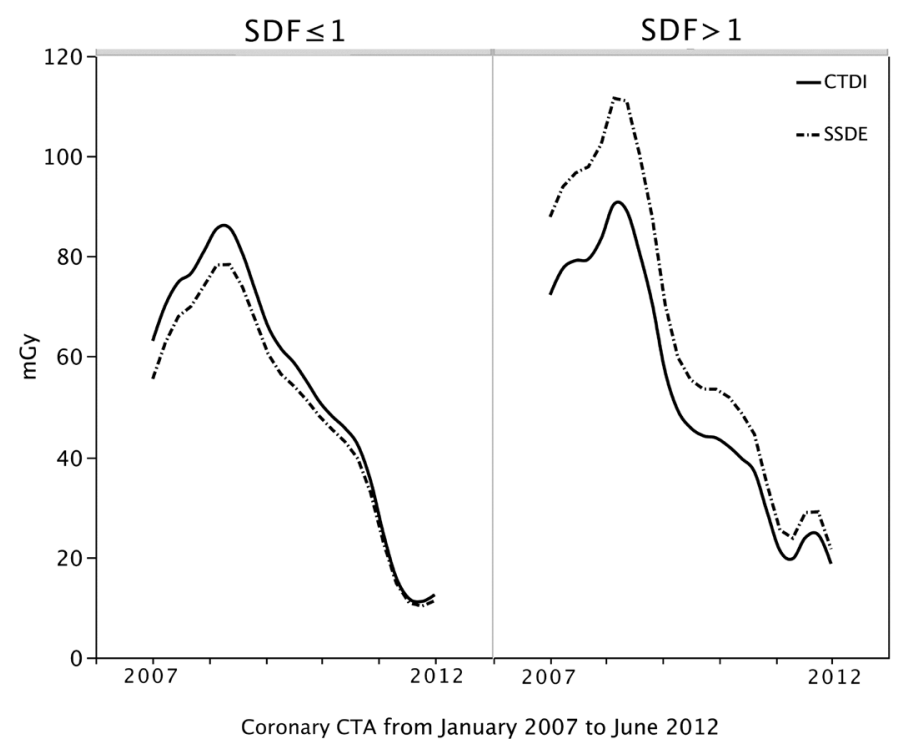

Figure 3. Temporal trends of CTDI compared with SSDE in patients with SDF $\leq 1$ (left panel) and in patients with SDF $>1$ (right panel). $\mathrm{SDF}=$ size dependent factor. 
(3.2\% vs. $5.4 \%(p=N S)$ and $12 \%$ vs. $21.5 \%(p=N S)$, respectively). This may be explained because the axial technique with prospective ECG gating requires a stabilization of heart rate below the $65 \mathrm{bpm}$ [17].

\section{Discussion}

Coronary CTA is a special practice, which involves radiological exposure of the patients, with wide inter-institutional and intra-institutional variability of the dose, and therefore requires strict monitoring to ensure the best quality assurance programs as well as quality control measures.

The European Directive also states that the Member status of the European Union should promote the estab-

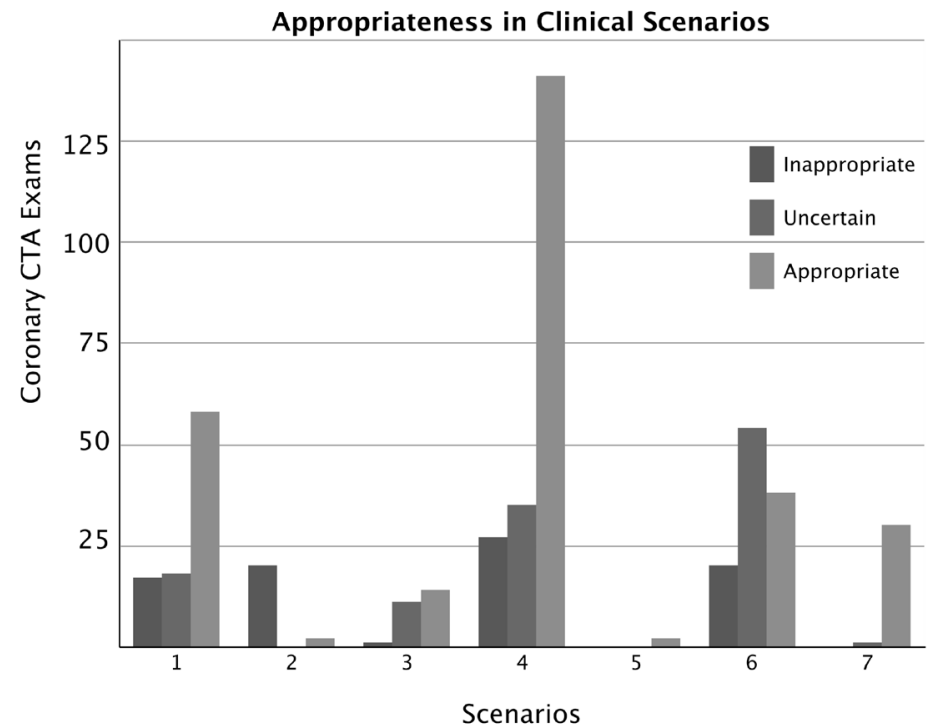

Figure 4. The graph shows the use of coronary CTA in the clinical scenarios according to guidelines (Table 1); the incidence of inappropriate, uncertain and appropriate exams in each scenario is also shown.

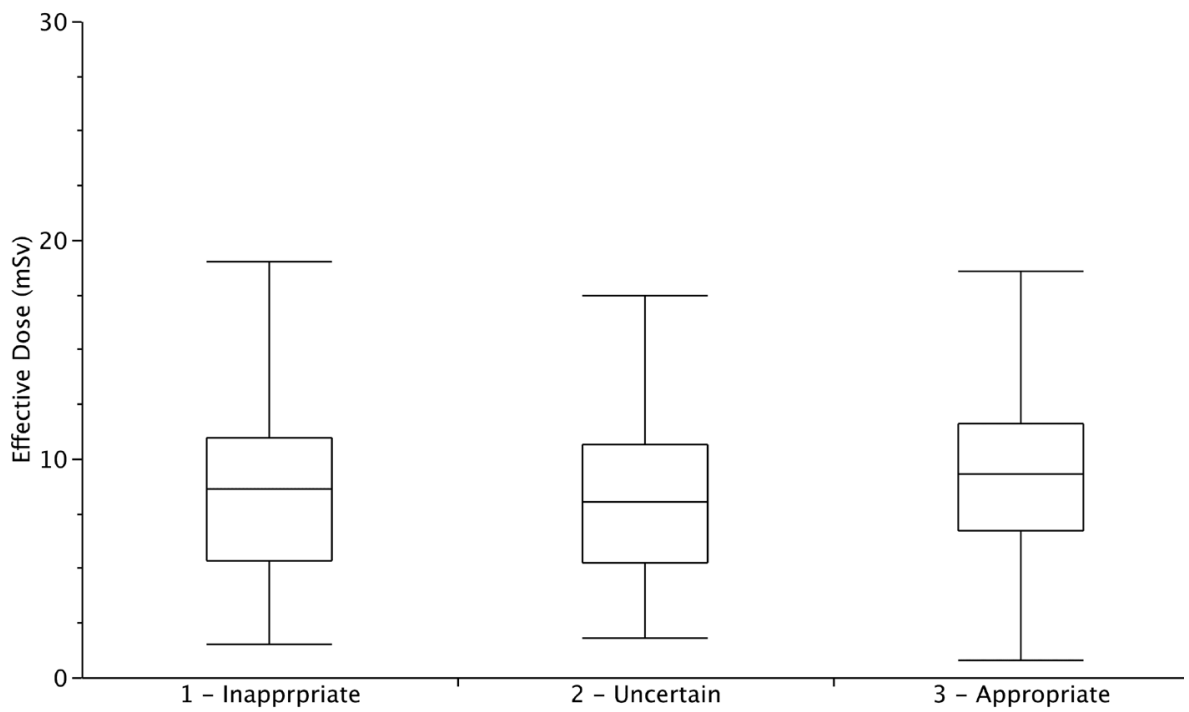

Appropriateness Level

Figure 5. Radiological dose was similar in the three groups of appropriate, partially appropriate and inappropriate indications. Data are referred to 489 patients enrolled between 2010 and 2012. 


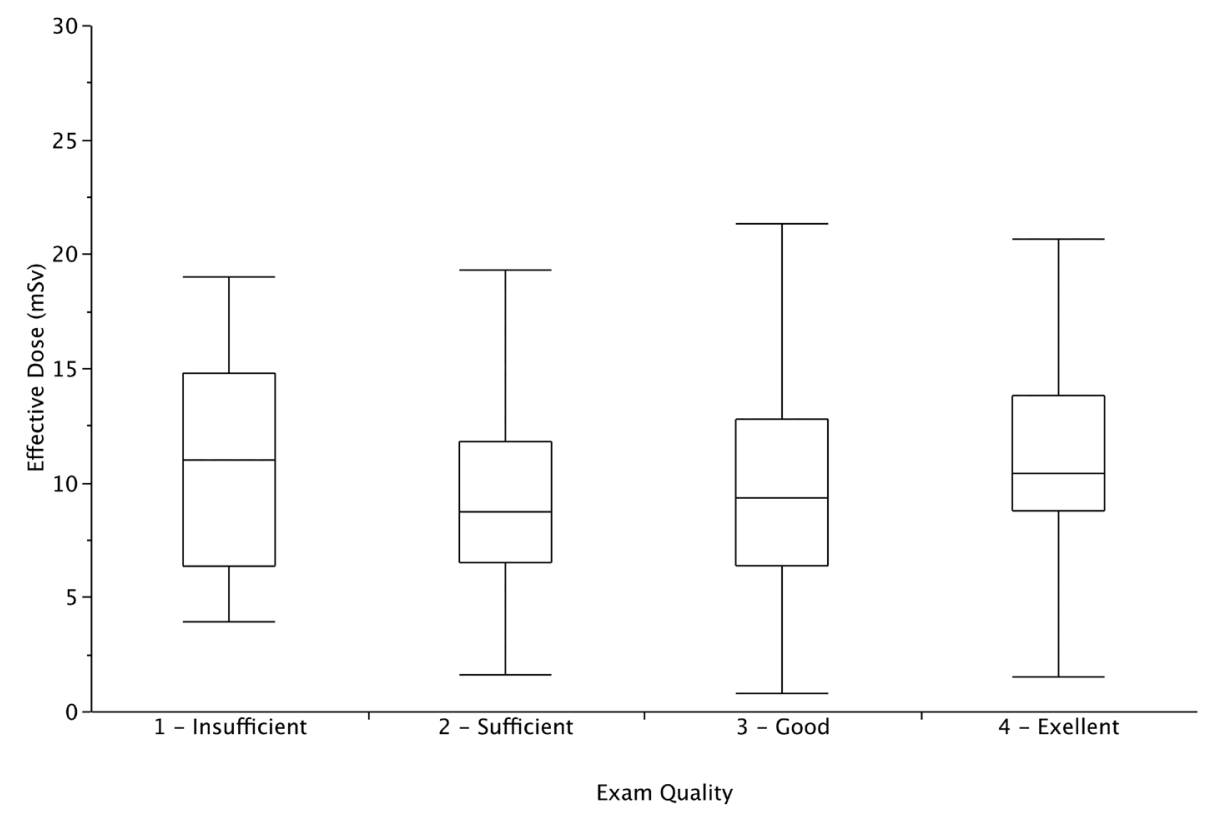

Figure 6. Radiological dose was similar in the four groups with excellent, good, sufficient and poor image quality. Data are referred to 489 patients enrolled between 2010 and 2012.

lishment and use of diagnostic reference levels for patient doses [18]. The availability of local, institution-specific, diagnostic reference levels are important for both patients and doctors. The patient should be informed and the patient's personal physician should keep records of the estimate of effective dose received. This recommendation is difficult to follow if patients' doses are not measured, archived and processed to detect high values of possible outliers. In addition, both in conventional diagnostic and interventional radiology examinations, the reference values are defined as indicators of good practice. If some hospitals are examining their patients using radiation doses consistently higher than reference values, they should revise their protocols to improve the situation. Our experience is fully consistent with this general rule. As radiation awareness increased, the need for audit of doses generated concern about doses that are relatively high compared with published benchmarks. This has led to greater focus by the operators on optimization, and change of technical parameters, steering towards the $100 \mathrm{kV}$ instead of the $120 \mathrm{kV}$ setting or by using axial prospective gating instead of the spiral retrospective gating technique. In the medium-term, this also led to resource reallocation to plan acquisition of dose-sparing CT, allowing dramatic (15-fold) reduction of doses [19].

The estimation of SSDE from CTDIvol improves the monitoring of the CT dose and might allow an appropriate tailoring of CT technique to patients' size and a more accurate control of radiation dose even in adult patient (Figure 3). However, at present, SSDE cannot be used for estimate effective dose and organ dose. Further studies will need for adjust SSDE evaluation and use it as a source for effective dose estimation [20].

The real-time assessment of appropriateness, using our electronic calculator, activate an interesting framework where an iterative knowledge-to-action cycle promoted an adaptation of knowledge to local context aiming to improve the rationale and appropriate use of Coronary CTA.

\section{Comparison with Previous Studies}

Concerns have been raised about how imaging facilities administer medical imaging exams that use radiation; wide variation has been observed among radiation doses associated with particular types of imaging examinations. For example, examining CT studies performed on adult patients within and across several institutions in the San Francisco Bay area, Smith-Bindam et al. report a mean 13-fold variation between the highest and the lowest dose for each type of study assessed [6]. Common interventional cardiology examinations show an, at least comparable, dose variability [21] [22]. In our 6 years of experience, the dose of single Coronary CTA ranged from 3474 to $55.39 \mathrm{mGy}^{*} \mathrm{~cm}$ with same examination, same type of machine, and operator team. The results of our study showing a clear trend towards dose reduction over time are consistent with data derived from 
two recent studies [23]-[25]. The first one is a systematic review of radiation dose associated with different generations of multidetector CTA in 66 studies published from 1998 to 2011; the second one is a single-center study; both showed that more dose-saving strategies were applied in recent CTA applications, including prospective ECG gating protocols, application of lower tube voltage and tube current modulation to achieve a noteworthy dose reduction. The third is a large multicenter study that reports DAP and image quality in a three-year period, but it do not let information about the exam appropriateness.

Currently in the literature there are no studies that have described a systematic prospective use of SSDE for Coronary CTA, so there is no information about the actual benefits.

The relatively high rate of examinations with inappropriate or uncertain indications is consistent with previous experience in the field of cardiovascular imaging, both with CT [26] or myocardial perfusion scintigraphy [27] and other highly specialized non-ionizing testing, such as stress echo [28].

\section{Study Limitation}

This is single-center experience, with a retrospective analysis. We monitored the experience of Coronary CTA from its very beginning, and therefore, the initial data may have mirrored a technical learning curve of operators. However, all of them had a large experience in cardiology, radiology and a previous dedicated training in the technique.

In this work we estimated SSDE retrospectively aiming to check the usefulness of this parameter for tailoring CT technique and dose at patient size. Thus, the radiation dose trends, described in this paper, are not influenced by SSDE estimation.

Effective dose was estimated from DLP. There is a lively debate concerning the merit of estimating effective dose from DLP for CT imaging and in particular for Coronary CTA [29] [30]. A fundamental of this debate is recognition of the purpose underlying any estimate of effective dose for medical exposures. Effective dose has been defined by the International Commission of Radiological Protection as a single dose quantity reflecting the overall risk to a reference person from any radiation exposure, where the risk is averaged over all ages and both sexes [30]. It is primarily used as a tool for assessing compliance with dose limits set to protect the public and workers, but it can also be useful for comparing the risks to a reference patient from different imaging techniques and procedures. The precision in reported values should be commensurate with the inherent substantial uncertainties. Within the framework of reference dosimetry for CT, broad coefficients of effective dose normalized to DLP can provide sufficiently precise estimates of effective dose for standard protocols and reference patients [16].

\section{Conclusion}

The findings of this study emphasize that awareness and audit are two tightly interconnected aspects of dose optimization, eventually leading to further dose sparing through technology upgrading and greater focus on appropriateness. The use of SSDE can allow individual technique optimization. Doses resulted similar in appropriate and inappropriate studies, and, less obviously, in those with good or poor image quality. The rate of inappropriate examinations is still too high even after dissemination of guidelines.

\section{Acknowledgements}

The study was made possible by the SUIT-Heart (Stop Useless Imaging Testing in Heart Disease) grant of the ITT-Istituto Toscano Tumori of the Region of Tuscany.

\section{References}

[1] Picano, E. (2004) Sustainability of Medical Imaging. Education and Debate. BMJ, 328, 578-580. http://dx.doi.org/10.1136/bmj.328.7439.578

[2] Mettler Jr., F.A., Thomadsen, B.R., Bhargavan, M., Gilley, D.B., Gray, J.E., Lipoti, J.A., McCrohan, J., Yoshizumi, T.T. and Mahesh, M. (2008) Medical Radiation Exposure in the U.S. in 2006: Preliminary Results. Health Physics, 95, 502-507. http://dx.doi.org/10.1097/01.HP.0000326333.42287.a2

[3] Cone, M. (2010) President’s Cancer Panel: Environmentally Caused Cancers Are “Grossly Underestimated” and 
“Needlessly Devastate American Lives”. Environmental Health News.

http://www.environmentalhealthnews.org/ehs/news/presidents-cancer-panel

[4] Malone, J., Guleria, R., Craven, C., Horton, P., Järvinen, H., Mayo, J., O’reilly, G., Picano, E., Remedios, D., Le Heron, J., Rehani, M., Holmberg, O. and Czarwinski, R. (2012) Justification of Diagnostic Medical Exposures: Some Practical Issues. Report of an International Atomic Energy Agency Consultation. British Journal of Radiology, 85, 523-538. http://dx.doi.org/10.1259/bjr/42893576

[5] Einstein, A.J., Henzlova, M.J. and Rajagopalan, S. (2007) Estimating Risk of Cancer Associated with Radiation Exposure from 64-Slice Computed Tomography Coronary Angiography. JAMA, 298, 317-323. http://dx.doi.org/10.1001/jama.298.3.317

[6] Smith-Bindman, R., Lipson, J., Marcus, R., Kim, K.P., Mahesh, M., Gould, R., Berrington de González, A. and Miglioretti, D.L. (2009) Radiation Dose Associated with Common Computed Tomography Examinations and the Associated Lifetime Attributable Risk of Cancer. Archives of Internal Medicine, 169, 2078-2086. http://dx.doi.org/10.1001/archinternmed.2009.427

[7] Bedetti, G., Botto, N., Andreassi, M.G., Traino, C., Vano, E. and Picano, E. (2008) Cumulative Patient Effective Dose in Cardiology. British Journal of Radiology, 81, 699-705. http://dx.doi.org/10.1259/bjr/29507259

[8] Ait-Ali, L., Andreassi, M.G., Foffa, I., Spadoni, I., Vano, E. and Picano, E. (2010) Cumulative Patient Effective Dose and Acute Radiation-Induced Chromosomal DNA Damage in Children with Congenital Heart Disease. Heart, 96, 269-274. http://dx.doi.org/10.1136/hrt.2008.160309

[9] Center for Devices and Radiological Health US Food and Drug Administration (2010) White Paper: Initiative to Reduce Unnecessary Radiation Exposure from Medical Imaging. http://www.fda.gov/Radiation-EmittingProducts/RadiationSafety/RadiationDoseReduction/ucm199994.htm

[10] Gerber, T.C., Carr, J.J., Arai, A.E., Dixon, R.L., Ferrari, V.A., Gomes, A.S., Heller, G.V., McCollough, C.H., McNittGray, M.F., Mettler, F.A., Mieres, J.H., Morin, R.L. and Yester, M.V. (2009) Ionizing Radiation in Cardiac Imaging: A Science Advisory from the American Heart Association Committee on Cardiac Imaging of the Council on Clinical Cardiology and Committee on Cardiovascular Imaging and Intervention of the Council on Cardiovascular Radiology and Intervention. Circulation, 119, 1056-1065. http://dx.doi.org/10.1161/CIRCULATIONAHA.108.191650

[11] Brindis, R. and Douglas, P.S. (2010) President's Page: The ACC Encourages Multi-Pronged Approach to Radiation Safety. Journal of the American College of Cardiology, 56, 522-524. http://dx.doi.org/10.1016/j.jacc.2010.07.001

[12] Amis Jr., E.S., Butler, P.F., Applegate, K.E., et al. (2007) American College of Radiology. American College of Radiology White Paper on Radiation Dose in Medicine. Journal of the American College of Radiology, 4, 272-284. http://dx.doi.org/10.1016/j.jacr.2007.03.002

[13] Taylor, A.J., Cerqueira, M., Hodgson, J.M., Mark, D., Min, J., O’Gara, P., Rubin, G.D., American College of Cardiology Foundation Appropriate Use Criteria Task Force; Society of Cardiovascular Computed Tomography; American College of Radiology; American Heart Association; American Society of Echocardiography; American Society of Nuclear Cardiology; North American Society for Cardiovascular Imaging; Society for Cardiovascular Angiography and Interventions; Society for Cardiovascular Magnetic Resonance, Kramer, C.M., Berman, D., Brown, A., Chaudhry, F.A., Cury, R.C., Desai, M.Y., Einstein, A.J., Gomes, A.S., Harrington, R., Hoffmann, U., Khare, R., Lesser, J., McGann, C., Rosenberg, A., Schwartz, R., Shelton, M., Smetana, G.W. and Smith Jr., S.C. (2010) ACCF/SCCT/ ACR/AHA/ASE/ASNC/NASCI/SCAI/SCMR 2010 Appropriate Use Criteria for Cardiac Computed Tomography: A Report of the American College of Cardiology Foundation Appropriate Use Criteria Task Force, the Society of Cardiovascular Computed Tomography, the American College of Radiology, the American Heart Association, the American Society of Echocardiography, the American Society of Nuclear Cardiology, the North American Society for Cardiovascular Imaging, the Society for Cardiovascular Angiography and Interventions, and the Society for Cardiovascular Magnetic Resonance. Journal of the American College of Cardiology, 56, 1864-1894. http://dx.doi.org/10.1016/j.jacc.2010.07.005

[14] American Association of Physicists in Medicine (2011) Size-Specific Dose Estimates (SSDE) in Pediatric and Adult Body CT Examinations: Report of AAPM Task Group 204. College Park.

[15] Halliburton, S.S., Abbara, S., Chen, M.Y., et al. (2011) SCCT Guidelines on Radiation Dose and Dose-Optimization Strategies in Cardiovascular CT. Journal of Cardiovascular Computed Tomography, 5, 198-224. http://dx.doi.org/10.1016/j.jcct.2011.06.001

[16] International Commission on Radiological Protection (2007) The 2007 Recommendations of the International Commission on Radiological Protection. ICRP, Publication no. 103. http://www.icrp.org/publications.asp

[17] Stolzmann, P., Goetti, R., Baumueller, S., Plass, A., Falk, V., Scheffel, H., Feuchtner, G., Marincek, B., Alkadhi, H. and Leschka, S. (2009) Prospective and Retrospective ECG-Gating for CT Coronary Angiography Perform Similarly Accurate at Low Heart Rates. European Journal of Radiology, 79, 85-91. http://dx.doi.org/10.1016/j.ejrad.2009.12.016

[18] European Commission. Directorate General Environment, Nuclear Safety and Civil Protection (2000) Radiation Pro- 
tection 116 Guidelines on Education and Training in Radiation Protection for Medical Exposures. Luxembourg.

[19] Raff, G.L., Chinnaiyan, K.M., Share, D.A., Goraya, T.Y., Kazerooni, E.A., Moscucci, M., Gentry, R.E. and Abidov, A. (2009) Advanced Cardiovascular Imaging Consortium Co-Investigators. Radiation Dose from Cardiac Computed Tomography before and after Implementation of Radiation Dose-Reduction Techniques. JAMA, 301, 2340-2348. http://dx.doi.org/10.1001/jama.2009.814

[20] Brink, J.A. and Morin, R.L. (2012) Size-Specific Dose Estimation for CT: How Should It Be Used and What Does It Mean? Radiology, 265, 666-668. http://dx.doi.org/10.1148/radiol.12121919

[21] Kocinaj, D., Cioppa, A., Ambrosini, G., Tesorio, T., Salemme, L., Sorropago, G., Rubino, P. and Picano, E. (2006) Radiation Dose Exposure during Cardiac and Peripheral Arteries Catheterisation. International Journal of Cardiology, 113, 283-284. http://dx.doi.org/10.1016/j.ijcard.2005.09.035

[22] Zorzetto, M., Bernardi, G., Morocutti, G. and Fontanelli, A. (1997) Radiation Exposure to Patients and Operators during Diagnostic Catheterization and Coronary Angioplasty. Catheterization and Cardiovascular Diagnosis, 40, 348351. http://dx.doi.org/10.1002/(SICI)1097-0304(199704)40:4<348::AID-CCD4>3.0.CO;2-9

[23] Sabarudin, A., Sun, Z. and Nq, K.H. (2012) A Systematic Review of Radiation Dose Associated with Different Generations of Multidetector CT Coronary Angiography. Journal of Medical Imaging and Radiation Oncology, 56, 5-17. http://dx.doi.org/10.1111/j.1754-9485.2011.02335.x

[24] Ghoshhajra, B.B., Engel, L.C., Major, G.P., Goehler, A., Techasith, T., Verdini, D., Do, S., Liu, B., Li, X., Sala, M., Kim, M.S., Blankstein, R., Prakash, P., Sidhu, M.S., Corsini, E., Banerji, D., Wu, D., Abbara, S., Truong, Q., Brady, T.J., Hoffmann, U. and Kalra, M. (2012) Evolution of Coronary Computed Tomography Radiation Dose Reduction at a Tertiary Referral Center. American Journal of Medicine, 125, 764-772. http://dx.doi.org/10.1016/j.amjmed.2011.10.036

[25] Chinnaiyan, K.M., Boura, J.A., Depetris, A., Gentry, R., Abidov, A., Share, D.A. and Raff, G.L. (2013) Progressive Radiation Dose Reduction from Coronary Computed Tomography Angiography in a Statewide Collaborative Quality Improvement Program: Results from the Advanced Cardiovascular Imaging Consortium. Circulation: Cardiovascular Imaging, 6, 646-654. http://dx.doi.org/10.1161/CIRCIMAGING.112.000237

[26] Ayyad, A.E., Cole, J., Syed, A., Desai, M.Y., Halliburton, S., Schoenhagen, P., Flamm, S.D. and Sola, S. (2009) Temporal Trends in Utilization of Cardiac Computed Tomography. Journal of Cardiovascular Computed Tomography, 3, 16-21. http://dx.doi.org/10.1016/j.jcct.2008.10.009

[27] Gibbons, R.J., Miller, T.D., Hodge, D., Urban, L., Araoz, P.A., Pellikka, P. and McKully, R.B. (2008) Application of Appropriateness Criteria to Stress Single Photon Emission Computed Tomography Sestamibi Studies and Stress Echocardiograms in an Academic Medical Center. Journal of the American College of Cardiology, 51, 1283-1289. http://dx.doi.org/10.1016/j.jacc.2007.10.064

[28] Picano, E., Pasanisi, E., Brown, J. and Marwick, T.H. (2007) A Gatekeeper for the Gatekeeper: Inappropriate Referrals to Stress Echocardiography. American Heart Journal, 154, 285-290. http://dx.doi.org/10.1016/j.ahj.2007.04.032

[29] Hurwitz, L.M., Reiman, R.E., Yoshizumi, T.T., Goodman, P.C., Toncheva, G., Nguyen, G. and Lowry, C. (2007) Radiation Dose from Contemporary Cardiothoracic Multidetector CT Protocols with an Anthropomorphic Female Phantom: Implications for Cancer Induction. Radiology, 245, 742-750. http://dx.doi.org/10.1148/radiol.2453062046

[30] Shrimpton, P.C., Wall, B.F., Yoshizumi, T.T., Hurwitz, L.M. and Goodman, P.C. (2009) Effective Dose and DoseLength Product in CT. Radiology, 250, 604-605. http://dx.doi.org/10.1148/radiol.2502081340 